\title{
Impact of obstructive sleep apnoea on insulin resistance in nonobese and obese children
}

\author{
Dorit Koren ${ }^{1,2}$, David Gozal ${ }^{1,3}$, Mona F. Philby ${ }^{1}$, Rakesh Bhattacharjee ${ }^{1,3,4}$ and \\ Leila Kheirandish-Gozal ${ }^{1}$
}

Affiliations: 'Sleep Medicine Section, Dept of Pediatrics, Pritzker School of Medicine, Biological Sciences Division, The University of Chicago, Chicago, IL, USA. ${ }^{2}$ Endocrinology and Metabolism Section, Dept of Pediatrics, Pritzker School of Medicine, Biological Sciences Division, The University of Chicago, Chicago, IL, USA. ${ }^{3}$ Pulmonology Section, Dept of Pediatrics, Pritzker School of Medicine, Biological Sciences Division, The University of Chicago, Chicago, IL, USA. ${ }^{4}$ Division of Respiratory Medicine, Dept of Pediatrics, University of California San Diego, San Diego, CA, USA.

Correspondence: Leila Kheirandish-Gozal, Section of Pediatric Sleep Medicine, Dept of Pediatrics, Pritzker School of Medicine, Biological Sciences Division, The University of Chicago, 5841 S. Maryland Avenue, Office C-113/MC2117, Chicago, IL 60637-1470, USA. E-mail: Igozalapeds.bsd.uchicago.edu

ABSTRACT Obstructive sleep apnoea (OSA) has been inconsistently associated with insulin resistance and adverse metabolic states. We aimed to assess independent contributions of OSA to insulin resistance and dyslipidaemia in a large paediatric cohort.

Habitually snoring children underwent overnight polysomnography, anthropometric measurements and fasting laboratory evaluations. Primary outcome measures included insulin, glucose, homeostasis model of insulin resistance, lipoproteins and sleep disturbance measures.

Among 459 children aged 5-12 years, obesity was the primary driver of most associations between OSA and metabolic measures, but sleep duration was inversely associated with glucose levels, with N3 and rapid eye movement (REM) sleep being negatively associated and sleep fragmentation positively associated with insulin resistance measures. In children with mild OSA, the presence of obesity increased the odds for insulin resistance, while higher apnoea/hypopnoea index values emerged among obese children who were more insulin-resistant.

The exclusive presence of interactions between OSA and obesity in the degree of insulin resistance is coupled with synergistic contributions by sleep fragmentation to insulin resistance in the context of obesity. Insufficient N3 or REM sleep may also contribute to higher glycaemia independently of obesity. Studies are needed to better delineate the roles of puberty and sleep fragmentation in insulin resistance and the metabolic syndrome.

@ERSpublications

OSA and obesity jointly affect insulin resistance, but insulin resistance is absent in nonobese children with OSA http://ow.ly/W4BmY

For editorial comment see Eur Respir J 2016; 47: 1050-1053 [DOI: 10.1183/13993003.00115-2016].

This article has supplementary material available from erj.ersjournals.com

Received: Aug 272015 | Accepted after revision: Nov 302015 | First published online: Feb 042016

Support statement: This study was supported by National Institutes of Health grant HL-65270. Funding information for this article has been deposited with FundRef.

Conflict of interest: None declared.

Copyright OERS 2016 


\section{Introduction}

Paediatric obesity prevalence has increased in recent decades [1], with a concomitant increase in obesity-related comorbidities, including type 2 diabetes mellitus (T2DM) [2] and the metabolic syndrome [3]. While weight loss can reduce the risk of developing obesity-related comorbidities [4], many find weight loss maintenance difficult [5]. Thus, it is imperative to identify other modifiable risk factors.

One potential candidate for intervention is obstructive sleep apnoea (OSA) syndrome, a common comorbidity of obesity [6] characterised by repetitive upper airway collapse during sleep, oxyhaemoglobin desaturation and recurrent arousals from sleep, i.e. sleep fragmentation [7]. The metabolic effects of paediatric OSA have been previously explored only in a relatively restricted number of studies, with somewhat inconsistent findings. In some studies, paediatric OSA has been shown to associate with visceral adiposity [8] and insulin resistance [9, 10]; however, another study found that insulin resistance was primarily determined by obesity, not OSA [11]. Given these contradictory results, we set out to prospectively study a large cohort of snoring children, and examine the independent contribution of OSA to insulin resistance and dyslipidaemia.

\section{Materials and methods}

\section{Subjects}

This was an observational study of healthy snoring children, approved by the University of Louisville Human Research Committee (protocol 474.99) and the University of Chicago Institutional Review Board (protocol 09-115-B). Snoring children aged 5-12 years undergoing evaluation for suspected OSA were prospectively recruited. Children with genetic syndromes or chronic medical conditions (excepting well-controlled asthma on no controller medications) and children receiving medications potentially affecting sleep, insulin or glucose homeostasis, or lipids (e.g. systemic glucocorticoids within 1 month of the study) were excluded from participation. Informed consent and age-appropriate assent were obtained.

\section{Anthropometric measurements}

All participants underwent anthropometric measurements. An electronic scale assessed weight. Height was assessed using a wall-mounted stadiometer. Body mass index (BMI; $\mathrm{kg} \cdot \mathrm{m}^{-2}$ ) was calculated. Age- and sex-adjusted standard scores (z-scores) for BMI were calculated [12]. Study participants with BMI z-score $\geqslant 1.64$ (BMI $\geqslant 95$ th percentile) were deemed obese [13]; other children were classified as nonobese.

\section{Overnight polysomnography}

All participants underwent overnight polysomnography (PSG) at Kosair Children's Hospital (Louisville, KY, USA) or at the Pediatric Sleep Laboratory at the University of Chicago Medical Center (Chicago, IL, USA). Parameters recorded and digitised using commercially available software included: eight-channel electroencephalogram (EEG), chin and bilateral anterior tibial and forearm electromyograms, bilateral electro-oculogram, heart rate by ECG, chest and abdominal wall movement by semicalibrated respiratory inductance plethysmography with derivation of sum from Lisajou analysis, airflow monitoring via side-stream end-tidal capnography, breath-by-breath assessment of end-tidal carbon dioxide levels via capnography (BCi International, Waukesha, WI, USA), nasal pressure transducer (Braebon, Kanata, ON, Canada), oronasal thermistor, pulse oximetry (SET; Massimo, Irvine, CA, USA) with simultaneous recording of the pulse waveform for arterial oxygen saturation $\left(\mathrm{SpO}_{2}\right)$, and analogue output from a body position sensor were simultaneously monitored. All measures were digitised using a commercially available PSG system (Nihon Kohden, Tokyo, Japan). Sleep architecture, respiratory events and arousals were scored by the same investigators (L.K.G., R.B. and D.G.) serially at the two sites using standard paediatric criteria [14].

Apnoeas were defined as $\geqslant 90 \%$ decrement in airflow lasting at least two breaths and hypopnoeas were defined as $>50 \%$ decrement in nasal airflow accompanied by either a $3 \%$ desaturation or an EEG arousal from sleep lasting $\geqslant 3 \mathrm{~s}$. Arousals were defined as per the revised American Academy of Sleep medicine guidelines [14]. Mean and nadir $\mathrm{SpO}_{2}$ were assessed. Children with an obstructive apnoea/hypopnoea index (AHI), defined as number of apnoeas and hypopnoea per hour of sleep, of $<1.0$ events $\cdot \mathrm{h}^{-1}$ total sleep time (TST) were deemed to have normal breathing during sleep, children with AHI $1-5$ events $\cdot \mathrm{h}^{-1}$ TST were considered to have mild OSA, and children with AHI $\geqslant 5$ events $\mathrm{h}^{-1}$ TST were considered to have moderate-severe OSA [11].

\section{Metabolic parameters}

The morning following the sleep study, participants had fasting blood drawn. Fasting plasma insulin (FPI) levels were measured using a radioimmunoassay kit (Coat-A-Count Insulin; Diagnostics Products, now Siemens Medical Solutions Diagnostics, assay no longer manufactured), sensitivity $1.2 \mu \mathrm{IU} \cdot \mathrm{mL}^{-1}$, and subsequently using a solid-phase, two-site chemiluminescent immunometric assay (Immulite 2000; Siemens Medical Solutions Diagnostics, Los Angeles, CA, USA), measuring range $2-300 \mu \mathrm{IU} \cdot \mathrm{mL}^{-1}$. Of note, the 
initial insulin assay was more sensitive, allowing insulin values between 1.2 and $2 \mu \mathrm{IU} \cdot \mathrm{mL}^{-1}$ to be determined; children with equivalent insulin sensitivity with insulin measured using the second assay would have insulin values reported as $<2 \mu \mathrm{IU} \cdot \mathrm{mL}^{-1}$, meaning homeostasis model assessment of insulin resistance (HOMA-IR) and the McAuley index could not be calculated or mean HOMA-IR values for the group measured with the second insulin assay. To avoid introducing a potential bias between children whose insulin levels were measured with the two different methodologies due to measurement sensitivity differences, any child with FPI $<2.0 \mu \mathrm{IU} \cdot \mathrm{mL}^{-1}$ was excluded from analyses. Fasting plasma glucose (FPG) was measured using a hexokinase glucose 6-phosphate dehydrogenase method (Flex Reagent Cartridges; Dade Behring, Newark, DE, USA) and using a UV enzymatic method with hexokinase (Roche Cobas 8000 702 platform; Roche, Basel, Switzerland); FPG levels $<63 \mathrm{mg} \cdot \mathrm{dL}^{-1}$ were excluded from analyses as artefactual (likely due to excessive specimen processing time). Children with FPG values consistent with T2DM $\left(>125 \mathrm{mg} \cdot \mathrm{dL}^{-1}\right.$ ) were excluded from analyses, as they constitute a separate metabolic disease population.

High-density lipoprotein (HDL), low-density lipoprotein (LDL), total cholesterol and triglycerides were measured using homogenous enzymatic colorimetric assays (Roche Cobas 8000502 platform). Analytical measuring range and assay coefficients of variations (CVs) were: 1) total cholesterol: range 3.86$800 \mathrm{mg} \cdot \mathrm{dL}^{-1}, \mathrm{CV} \leqslant 1.6 \%$; 2) HDL cholesterol: range $3-120 \mathrm{mg} \cdot \mathrm{dL}^{-1}, \mathrm{CV} \leqslant 1.5 \%$; 3) LDL cholesterol: range $3.86-548 \mathrm{mg} \cdot \mathrm{dL}^{-1}$, range $\leqslant 2.7 \%$; and 4) triglycerides: range $8.85-885 \mathrm{mg} \cdot \mathrm{dL}^{-1}, \mathrm{CV} \leqslant 2.0 \%$. Total cholesterol/HDL, LDL/HDL and triglyceride/HDL ratios were calculated. Higher values for total cholesterol/HDL [15], LDL/HDL [16] and triglyceride/HDL [17] ratios are associated with increased cardiovascular disease risk.

\section{Calculated insulin sensitivity parameters}

HOMA-IR was calculated as: $\left(\right.$ FPI $\left.\left(\mu \mathrm{IU} \cdot \mathrm{mL}^{-1}\right) \times \mathrm{FPG}\left(\mathrm{mg} \cdot \mathrm{dL}^{-1}\right)\right) / 405[18,19]$. HOMA-IR values $>2.5$ were considered "abnormal" (i.e. consistent with insulin resistance) [20].

The McAuley index, which incorporates a weighted combination of FPI and triglycerides [21], was calculated as: $\exp \left(2.63-0.28 \times \ln \left(\mathrm{FPI}\left(\mu \mathrm{IU} \cdot \mathrm{mL}^{-1}\right)-0.31 \times \ln \left(\right.\right.\right.$ triglycerides $\left.\left(\mathrm{mmol} \cdot \mathrm{L}^{-1}\right)\right)$. Lower values denote greater insulin resistance.

\section{Statistical analyses}

Statistical analyses were performed using SPSS version 22.0 (IBM, Armonk, NY, USA). Skewed variables were natural log-transformed to normalise distributions. Correlation analyses were used to examine associations between OSA measures and anthropometric and metabolic variables. Stepwise multivariate linear regression models were constructed to assess relationships between sleep measures (e.g. TST or $\mathrm{AHI}$ ) and metabolic variables (e.g. FPI) while controlling for BMI and, if significantly associated on correlation analyses, age. A p-value $<0.05$ was used as the cut-off for statistical significance. No differences emerged between the two study sites; as such, the data were merged and the cohort analysed as one group. As obese participants could be more susceptible to OSA's metabolic sequelae, and as severe versus mild OSA could have a differential metabolic impact, subjects were divided into three groups of OSA severity, and subdivided into nonobese and obese within OSA groups. Chi-squared tests were used to compare categorical characteristics, while ANOVA or Kruskal-Wallis tests, as appropriate, were used to compare continuous metabolic and sleep characteristics among the groups.

To further assess the impact of OSA upon insulin resistance, we compared sleep measures among children at extremes of insulin sensitivity. Children with intermediate insulin sensitivity were excluded from this stage of the analysis in order to see whether more subtle differences in sleep architecture and sleep disturbances between children with greatest and lowest insulin sensitivity would emerge. HOMA-IR quartiles were calculated for the entire study population and children in the lowest and highest quartiles (quartile 1, HOMA-IR <0.91; quartile 4, HOMA-IR >2.33) within each AHI and BMI group were included in a further subanalysis. t-tests or Mann-Whitney U-tests were performed to compare differences in OSA elements, age and degree of obesity between children in the lowest versus highest HOMA-IR quartiles within each subgroup.

\section{Results}

\section{Study population}

537 children completed the study. Of these, two children were excluded for FPG $>125 \mathrm{mg} \cdot \mathrm{dL}^{-1}, 13$ were excluded for FPG $<63 \mathrm{mg} \cdot \mathrm{dL}^{-1}$ and 62 were excluded for insulin levels $<2 \mu \mathrm{IU} \cdot \mathrm{mL}^{-1}$. Thus, 460 children were included in the final cohort: mean age $7.2 \pm 1.2$ years, 58\% male, 167 obese, $59 \%$ Caucasian, $33 \%$ African-American, $6 \%$ Hispanic and $2 \%$ of other racial or ethnic origins. Baseline demographic characteristics by subgroup are shown in table 1. Demographic comparisons showed no significant differences in age, sex, race/ethnicity or height across OSA groups, or between nonobese and obese 
TABLE 1 Demographics and anthropometrics: comparisons between nonobese and obese apnoea/hypopnoea index (AHI) groups

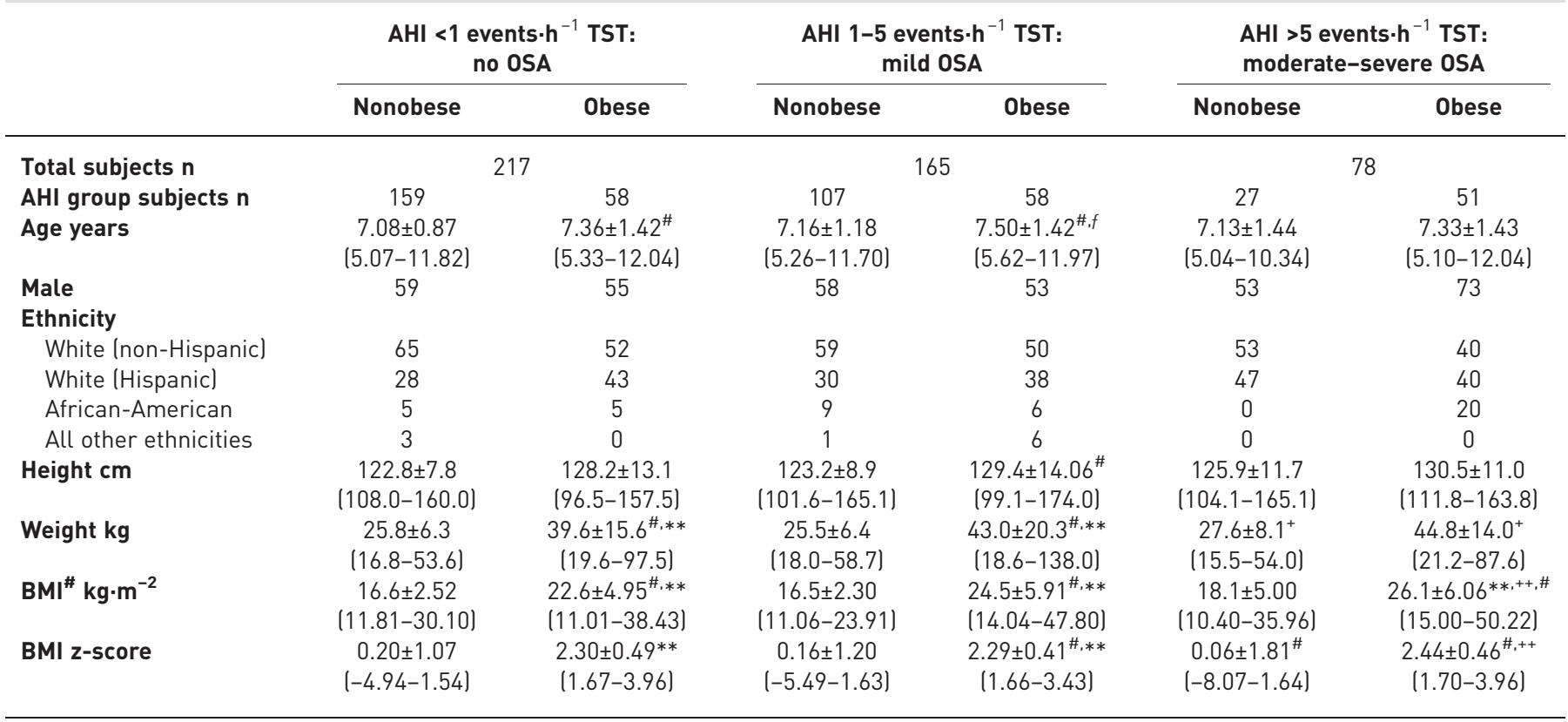

Data are presented as $\%$ or mean \pm SD (range), unless otherwise stated. TST: total sleep time; OSA: obstructive sleep apnoea; BMI: body mass index. ${ }^{\#}$ : nonnormal distribution: nonparametric testing. ${ }^{* *}: p<0.01 ;{ }^{f}: 0.05<p<0.1$ for t-test/Mann-Whitney comparisons between nonobese and obese within an AHI group. ${ }^{+}$: $p<0.05 ;{ }^{++}$: $p<0.01$ for ANOVA/Kruskal-Wallis comparisons between nonobese subjects across AHI groups or obese subjects across AHI groups.

participants within OSA groups. BMI and BMI z-score differed significantly only in obese children across AHI groups, with highest values seen in moderate-severe OSA. 53\% of all study participants had OSA and $17 \%$ had moderate-severe OSA (AHI $>5$ events $\cdot \mathrm{h}^{-1} \mathrm{TST}$ ). The prevalence and severity of OSA were higher in nonobese versus obese participants; $65 \%$ of obese participants had some degree of OSA and $31 \%$ had moderate-severe OSA, while $46 \%$ of nonobese participants had some degree of OSA and only $9 \%$ had moderate-severe OSA.

\section{Metabolic parameters}

Age was positively associated with BMI (correlation coefficient (p-value) $0.207(<0.0005)$, BMI z-score (0.099 (0.035)), FPI (0.111 (0.018)) and HOMA-IR (0.116 (0.013)), and negatively with the McAuley index $(-0.113(0.016))$. Similarly, BMI was positively associated with FPI $(0.093$ (0.045)), HOMA-IR (0.099 $(0.033))$ and triglycerides $(0.096(0.041))$, and negatively with the McAuley index $(0.124(0.0008))$, while BMI z-score was significantly negatively associated with the McAuley index only $(-0.091(0.050))$; glucose levels trended to associate positively with age and BMI, while remaining lipoprotein levels were not associated with age, BMI or BMI z-score (data not shown). These results showed that older and more obese children were more likely to be insulin resistant.

Correlation analyses examining associations between sleep variables and anthropometric and metabolic outcomes are shown in tables 2 and 3 and, for lipids, in supplementary table S1. AHI, apnoea index, obstructive apnoea index and all arousal indices (ArIs) were significantly positively associated with BMI and BMI z-score (spontaneous and total ArIs only were associated with age), and nadir and mean $\mathrm{SpO}_{2}$ associated negatively with BMI and BMI z-score, i.e. more severe OSA accompanied greater obesity. The spontaneous and total ArIs were associated positively with FPI and HOMA-IR, and negatively with the McAuley index, suggesting greater insulin resistance in those with more fragmented sleep; the total ArI also associated positively with FPG and triglycerides. FPG, FPI and lipoprotein levels were not associated with apnoea indices or $\mathrm{SpO}_{2}$. In support of these findings, the proportions of rapid eye movement (REM) sleep and delta wave sleep (stage N3) were also significantly but modestly associated with FPI; percentage TST in REM also associated modestly with HOMA-IR. TST was strongly negatively associated with FPG (as well as with age, BMI and BMI z-score). Percentage TST in N3 was significantly negatively associated with FPG. No other sleep architecture measure associated significantly with any metabolic outcome examined. 
TABLE 2 Associations between obstructive sleep apnoea measures and anthropometric and metabolic parameters in entire study population

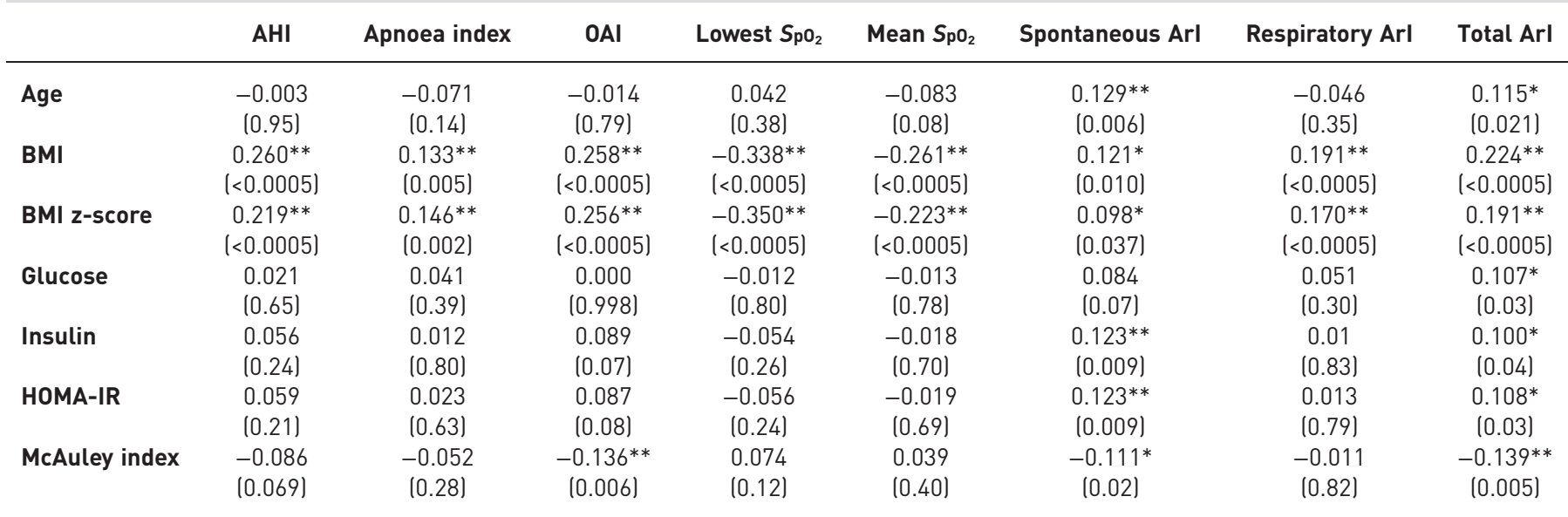

Data are presented as Spearman's rho correlation coefficient ( $p$-value). AHI: apnoea/hypopnoea index; OAl: obstructive apnoea index; SpO ${ }_{2}$ arterial oxygen saturation measured by pulse oximetry; Arl: arousal index; BMI: body mass index; HOMA-IR: homeostasis model assessment of insulin resistance. ${ }^{*}: p<0.05 ;{ }^{* *}: p<0.01$.

Comparisons of metabolic measures between nonobese and obese children within OSA groups and among nonobese and obese across OSA groups are depicted in table 4 and supplementary table S2. No significant metabolic differences were seen between either nonobese children or obese children with varying degrees of OSA. The McAuley index was significantly lower in obese versus nonobese children with moderatesevere OSA, indicating greater insulin resistance in the obese population; obese children without OSA and with mild OSA demonstrated no significant metabolic differences compared with nonobese children, although trends were seen towards lower higher FPI and triglycerides in obese versus nonobese children with mild OSA (figure 1).

The distribution of OSA measures is depicted in table 5. AHI differed among the groups by definition. Comparing sleep parameters across nonobese and across obese children with varying degrees of OSA, those with moderate-severe OSA had significantly lower $\mathrm{SpO}_{2}$ values, more sleep fragmentation due to respiratory events and higher sleep pressure score (a surrogate measure of sleep fragmentation [22]). Comparing sleep disturbance measures between nonobese and obese children within OSA subgroups, we found no differences

TABLE 3 Associations between sleep architecture and anthropometric and metabolic parameters in entire study population

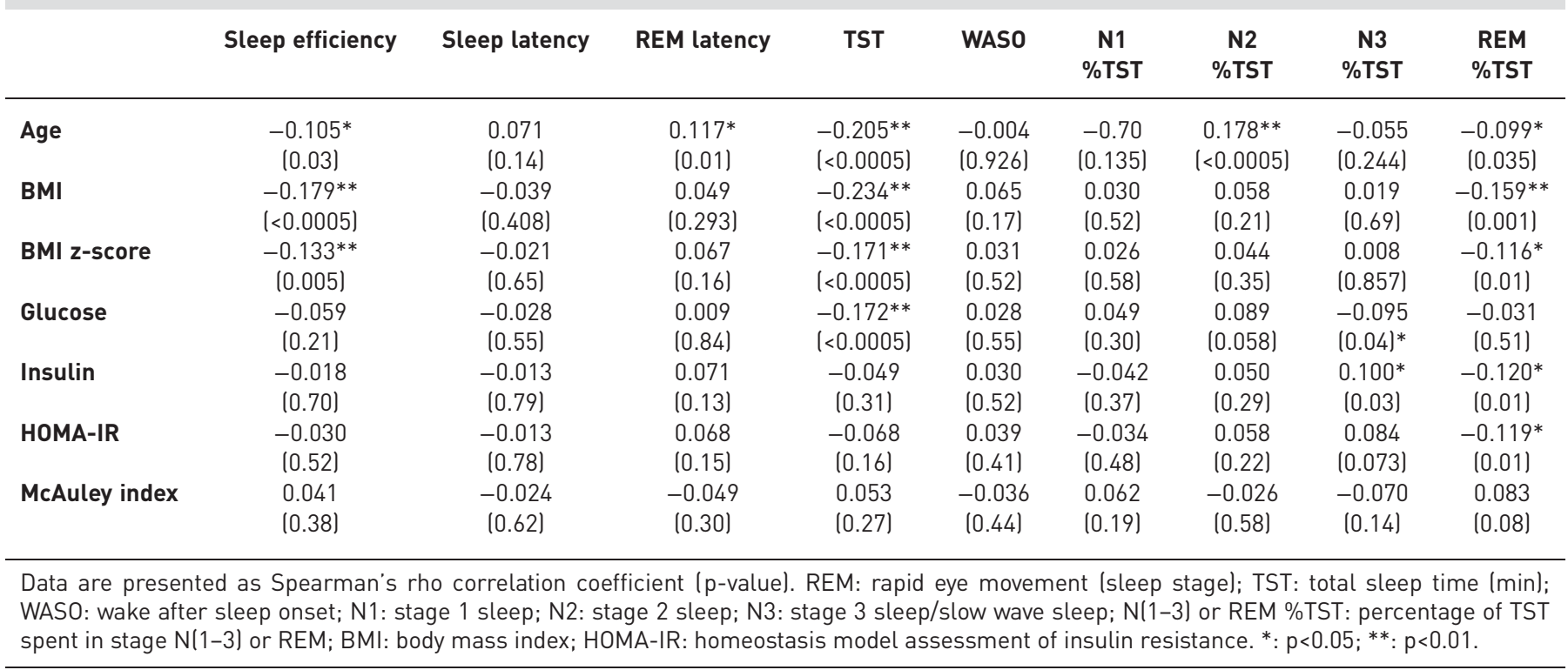


TABLE 4 Comparison of metabolic parameters across nonobese and obese apnoea/hypopnoea index (AHI) groups

\begin{tabular}{|c|c|c|c|c|c|c|}
\hline & \multicolumn{2}{|c|}{$\begin{array}{c}\text { AHI }<1 \text { events } \cdot \mathrm{h}^{-1} \text { TST: } \\
\text { no OSA }\end{array}$} & \multicolumn{2}{|c|}{$\begin{array}{c}\text { AHI 1-5 events.h }{ }^{-1} \text { TST: } \\
\text { mild OSA }\end{array}$} & \multicolumn{2}{|c|}{$\begin{array}{l}\text { AHI }>5 \text { events } \cdot h^{-1} \text { TST: } \\
\text { moderate-severe OSA }\end{array}$} \\
\hline & Nonobese & Obese & Nonobese & Obese & Nonobese & Obese \\
\hline $\mathrm{AHI}$ group subjects & 161 & 58 & 107 & 58 & 27 & 51 \\
\hline FPG $\mathrm{mg} \cdot \mathrm{dL}^{-1}$ & $87.5 \pm 8.48$ & $88.6 \pm 7.40$ & $87.1 \pm 7.16$ & $87.9 \pm 6.75$ & $90.9 \pm 10.22^{\#}$ & $88.5 \pm 10.28$ \\
\hline $\mathrm{FPI} \mu \mathrm{IU} \cdot \mathrm{mL}^{-1 \#}$ & $8.50 \pm 6.95$ & $9.71 \pm 9.22$ & $8.00 \pm 4.63$ & $10.85 \pm 11.43^{f}$ & $8.20 \pm 6.35$ & $8.28 \pm 4.76$ \\
\hline Abnormal HOMA-IR \%" & 24.2 & 23.7 & 18 & 28 & 33 & 41 \\
\hline McAuley index & $9.54 \pm 2.42$ & $9.43 \pm 2.41$ & $9.39 \pm 2.33$ & $8.81 \pm 2.42$ & $9.38 \pm 2.16$ & $8.81 \pm 1.95^{* *}$ \\
\hline
\end{tabular}

Data are presented as $n$ or mean $\pm S D$, unless otherwise stated. TST: total sleep time; OSA: obstructive sleep apnoea; FPG: fasting plasma glucose; FPI: fasting plasma insulin; In: natural log; HOMA-IR: homeostasis model assessment of insulin resistance. ${ }^{\text {: }}$ : nonnormal distribution: nonparametric testing; ": percentage of children in each subgroup with "abnormal" HOMA-IR values $>2.5$ (i.e. consistent with insulin resistance). ${ }^{* *}: p<0.01 ;{ }^{f}: 0.05<p<0.1$ for t-test/Mann-Whitney comparisons between nonobese and obese within an AHI group.

between nonobese and obese children without OSA, lower mean and nadir $\mathrm{SpO}_{2}$ levels in obese versus nonobese children with mild OSA, and significantly higher AHI, respiratory ArI, and lower mean and nadir $\mathrm{SpO}_{2}$ in obese versus nonobese children. The differences in mean AHI (19.4 versus 11.2) and nadir $\mathrm{SpO}_{2}(79.1$ versus $87.4 \%$ ) were also of considerable clinical significance. In other words, even within the moderatesevere OSA group, the obese children had greater disease severity than the nonobese children.

HOMA-IR quartile analyses are presented in supplementary table S3. Children in HOMA-IR quartile 4 (most insulin resistant) versus quartile 1 (least insulin resistant) had higher BMI and/or BMI z-score in all subgroups examined except nonobese children without OSA and obese children with moderate-severe OSA. In obese children with mild OSA, the AHI was significantly higher in quartile 4 versus quartile 1 (2.4 \pm 0.9 versus $1.7 \pm 0.9)$. In obese children with moderate-severe OSA, the respiratory ArI was near-significantly greater in those in quartile 4 versus quartile $1(25.7 \pm 14.1$ versus $8.1 \pm 5.2, \mathrm{p}=0.053)$, clinically a very significant difference (figure 2).

On multivariate linear regression analyses (table 6), spontaneous ArI significantly predicted FPI and HOMA-IR, albeit less than BMI. Total ArI was the strongest predictor of the McAuley index, with trends towards contributions from BMI and age. Finally, total sleep duration was the strongest contributor of FPG and HDL, with no contribution from either age or BMI. No other metabolic parameter was predicted by either OSA or sleep architecture measures after including BMI and/or age in regression modelling (data not shown).

FIGURE 1 McAuley index. Data represent mean \pm SD. Comparisons were made across the groups. *: $p<0.05$ for comparison between nonobese and obese participants with the apnoea/hypopnoea index $\left(\mathrm{AHI}\right.$; events $\left.\cdot \mathrm{h}^{-1}\right)$ group.

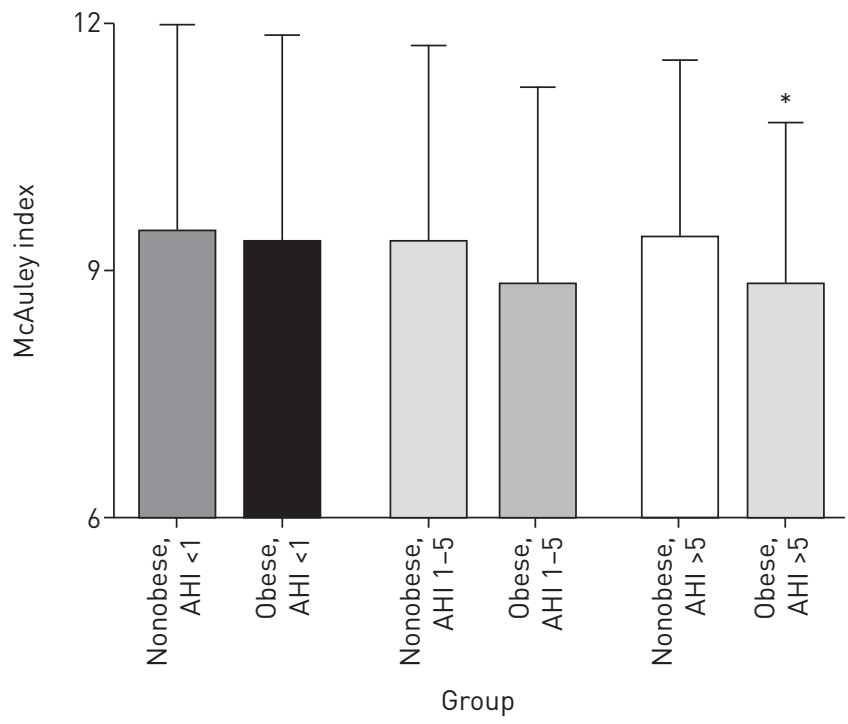


TABLE 5 Obstructive sleep apnoea (OSA) elements results across nonobese and obese apnoea/hypopnoea index (AHI) groups

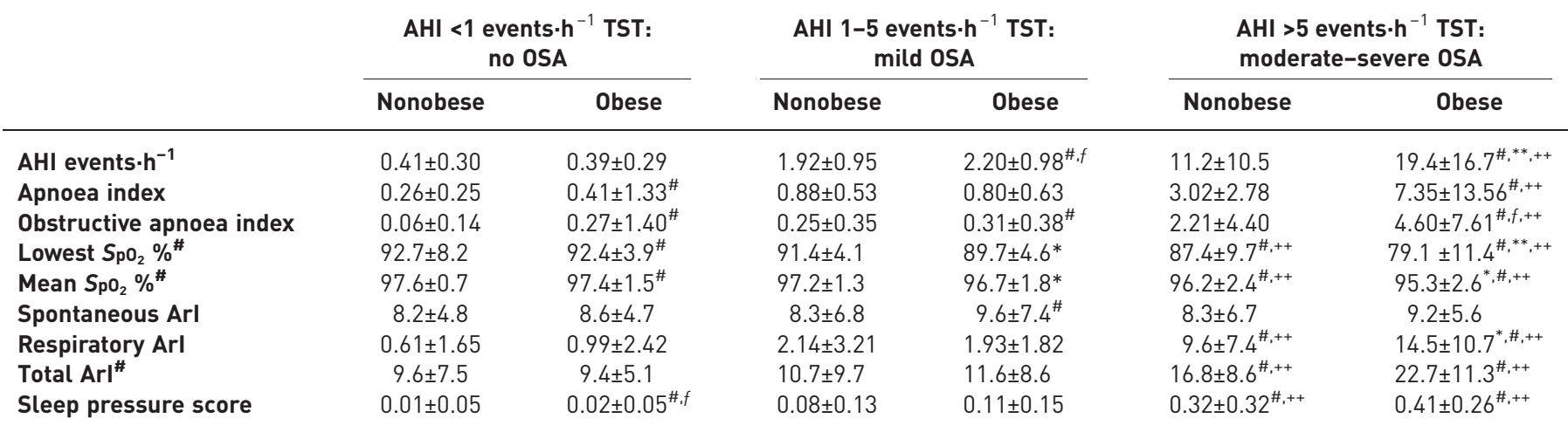

Data are presented as mean \pm SD. TST: total sleep time; $\mathrm{SpO}_{2}$ : arterial oxygen saturation measured by pulse oximetry; Arl: arousal index. \#: nonnormal distribution: nonparametric testing. ${ }^{*}$ : $p<0.05$; ${ }^{* *}: p<0.01 ;{ }^{f}: 0.05<p<0.1$ for t-test/Mann-Whitney U-test comparisons between nonobese and obese within an AHI group. ${ }^{++}: \mathrm{p}<0.01$ for ANOVA/Kruskal-Wallis comparisons between nonobese subjects across AHI groups or obese subjects across $\mathrm{AHI}$ groups.

\section{Discussion}

This study represents the largest cohort published to date examining relationships between OSA, sleep architecture and indicators of paediatric insulin resistance and the metabolic syndrome. We found that relationships between insulin resistance and dyslipidaemia were predominantly determined by adiposity rather than OSA or sleep architecture, but that sleep fragmentation contributed independently to insulin resistance and that total sleep duration was the primary, negative predictor of glucose and (to a limited extent) HDL, independently of obesity or OSA. Subcohort analyses further revealed that: 1) children with severe versus mild-moderate OSA were more obese, and among children with moderate-severe OSA, obese versus nonobese children had more severe sleep-disordered breathing; 2) OSA presence or degree did not impact insulin resistance, FPG, lipoprotein profiles or ratios among nonobese or obese children; 3) in obese children with mild OSA, higher AHI was seen in those with greater insulin resistance; and 4) sleep fragmentation due to respiratory arousals trended towards being higher in more insulin resistance obese children with moderate-severe OSA. Taken together, our findings suggest that obesity rather than OSA is the primary determinant of insulin resistance and metabolic dysfunction in young children, although OSA may further predispose to insulin resistance in obese children, that sleep fragmentation may contribute to insulin resistance independently of obesity and that insufficient sleep may predispose to higher glucose levels independently of either adiposity or OSA.

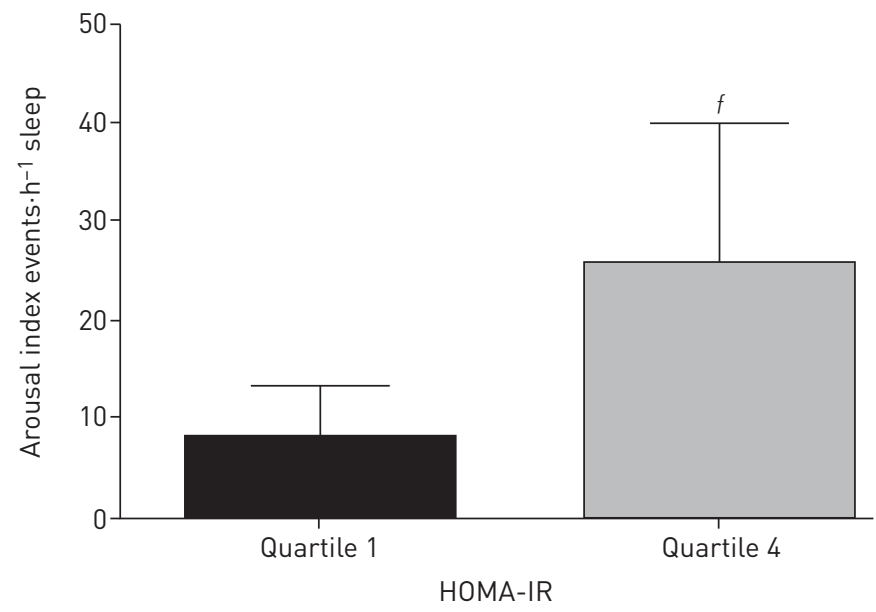

FIGURE 2 Respiratory arousal index. Data represent mean \pm SD. Comparisons were made across the groups. $f: 0.1<p<0.05$ for comparison between obese children with apnoea/hypopnoea index $>5$ events $\cdot h^{-1}$ in the lowest and highest homeostasis model assessment of insulin resistance (HOMA-IR) quartiles. 


\begin{tabular}{|c|c|c|c|c|}
\hline Model & $\begin{array}{l}\text { Variable } \\
\text { entered }\end{array}$ & $\begin{array}{c}\text { Variable standardised } \\
\beta \text {-coefficient }\end{array}$ & $\begin{array}{c}\text { B-coefficient } \\
\text { p-value }\end{array}$ & $\begin{array}{l}\text { Overall model } \\
\text { adjusted } \mathbf{R}^{2}\end{array}$ \\
\hline \multirow[t]{2}{*}{ Fasting insulin" } & BMI & 0.158 & 0.001 & 0.033 \\
\hline & Spontaneous Arl & 0.101 & 0.03 & \\
\hline \multirow[t]{2}{*}{ HOMA-IR } & BMI & 0.179 & $\leqslant 0.0005$ & 0.042 \\
\hline & Spontaneous Arl & 0.111 & 0.02 & \\
\hline \multirow[t]{3}{*}{ McAuley index } & Total Arl & -0.124 & 0.01 & 0.01 \\
\hline & Age & -0.094 & 0.06 & \\
\hline & BMI & -0.10 & 0.06 & \\
\hline Fasting glucose & TST & -0.186 & $\leqslant 0.0005$ & 0.032 \\
\hline HDL & TST & -0.098 & 0.04 & 0.007 \\
\hline
\end{tabular}

BMI: body mass index; Arl: arousal index; HOMA-IR: homeostasis model assessment of insulin resistance; TST: total sleep time; HDL: high-density lipoprotein. \#: natural log-transformed for nonnormal distribution.

Impact of sleep duration, architecture and disturbance on glycaemia insulin resistance in nonobese and obese children

We found that obesity rather than OSA was the primary correlate of insulin resistance, but in obese children with mild OSA, higher AHI values were seen in the highest versus lowest HOMA-IR quartiles. These findings suggest a possible mild contributory effect of OSA to insulin resistance development in the context of obesity and its a priori predisposition to metabolic derangements. Similarly, and in agreement with our previous studies, significant associations emerged between TST and glucose independently of OSA or obesity, and between measures of insulin resistance and the proportion of total sleep time spent in REM and N3 sleep stages, a finding that has also been replicated in adults with OSA [23, 24].

Our findings that OSA is inconsistently associated with insulin resistance in children stand in contrast to a significant body of adult literature, wherein OSA associates with both insulin resistance [25] and T2DM $[26,27]$, and add to a body of contradictory paediatric literature: some studies show that OSA confers an increased risk of adverse metabolic states $[9,28]$, while we and others have found either no association with insulin resistance [29] or associations only in obese children [30].

Several explanations for the inconsistency of paediatric studies vis-à-vis results in adults present themselves. OSA and obesity in children may have existed for too short a time for metabolic comorbidities of either to predictably and consistently develop. Alternatively, the explanation for contradictory results in the paediatric literature may reside in puberty. Most of the paediatric studies showing a positive association of OSA with insulin resistance and the metabolic syndrome studied adolescents and, in the case of KeLLY et al. [31], explicitly examined the contributory effect of puberty $[9,28,29,32]$. The divergent findings in pre-pubertal children versus adolescents may be multifactorial: fat mass [33] and, in boys, intra-abdominal fat [34] increase significantly during puberty, predisposing to insulin resistance. Also, paediatric OSA appears to have two different phenotypic variants, sometimes termed OSA type I and OSA type II [35]. OSA type I, which predominates in younger children, relates primarily to lymphadenoidal hypertrophy, while OSA type II, which predominates in adolescents, primarily develops in the context of obesity. These phenotypes present similarly, but likely confer differing predispositions to metabolic sequelae [35]. Thus, associations between OSA and insulin resistance and the metabolic syndrome in adolescents versus younger children should be carefully delineated in future studies.

\section{Sleep fragmentation and insulin resistance}

Examination of the entire cohort showed that sleep fragmentation due to spontaneous arousals contributed to insulin resistance separately from obesity. Incidentally, we found that the McAuley index was a more sensitive measure of sleep fragmentation's impact upon insulin resistance than HOMA-IR. Our findings support a growing body of literature suggesting that sleep fragmentation may independently contribute to insulin resistance, both in the context of OSA [30,36] and in healthy adults, where decreased insulin sensitivity and glucose disposal following experimental sleep fragmentation [37] suggest a causal link. Sleep fragmentation could impact insulin resistance through various potential mechanisms, including autonomic activation and increased cortisol levels [37]. Also, in murine models, we have found that experimental sleep fragmentation without hypoxaemia leads to visceral fat accumulation and insulin resistance [38, 39], accompanied by differentially expressed microRNA in a network of genes regulating insulin [40]. Sleep disruption should thus be minimised, especially in children at risk for insulin resistance. Future studies 
should be undertaken in children and adolescents with upper airway resistance syndrome (a condition of fragmented sleep without OSA) [41] to assess whether their insulin homeostasis is perturbed.

\section{Strengths and limitations}

Our study had a number of strengths. We had a very large cohort of children, allowing for subtle relationships to be explored, including the quartile analysis. Our study included a sizable number of both nonobese and obese subjects with and without OSA, allowing for determination of which relationships were dependent upon adiposity and which existed only in the presence of OSA. Additionally, OSA diagnosis and severity assessment was based upon gold standard in-laboratory full PSG.

Our study also had several limitations. All participants were referred for habitual snoring, introducing a possible referral bias. Also, we did not Tanner stage our participants and cannot rule out that some may have been pubertal. Furthermore, all metabolic measures examined reflected the fasting rather than post-prandial state. Additionally, as sleep duration in the sleep laboratory may not necessarily reflect home sleep duration, our findings of associations between sleep duration in the sleep laboratory and glycaemia are necessarily limited. While both HOMA-IR and the McAuley index have been validated against the gold standard insulin sensitivity measure from the hyperinsulinaemic-euglycaemic clamp technique, fasting insulin levels nonetheless reflect hepatic insulin sensitivity and gluconeogenesis; the association of OSA with skeletal muscle or adipose tissue insulin sensitivity and glucose disposal could not be assessed. 29 subjects had impaired fasting glucose, too few to analyse separately; separate associations could prevail in this subgroup. Also, we did not measure haemoglobin A1c, a measure of long-term glycaemic homeostasis, and cannot exclude the possibility that some subjects had hyperglycaemia not captured by fasting glucose. Finally, a number of subjects were excluded for low FPG or for FPI below assay sensitivity, forming an effective "floor" to the examination of associations between OSA and insulin resistance; more insulin-sensitive subjects may have been excluded. Finally, as this was not an intervention study, causality cannot be inferred.

\section{Conclusion}

In conclusion, we found that obesity rather than OSA seems to be the major driver of metabolic dysfunction in young children. OSA may have a small contribution, particularly in the context of obesity, and sleep fragmentation also appears to pose an additive risk for insulin resistance. We cannot rule out a small effect of OSA on metabolic function in nonobese children. Future studies are needed to better define the role of puberty in modulating OSA's adverse metabolic impact and the role of sleep fragmentation in induction of insulin resistance.

\section{Acknowledgements}

The authors would like to gratefully acknowledge Richa Kulkarni (Sleep Medicine Section, Dept of Pediatrics, Pritzker School of Medicine, Biological Sciences Division, The University of Chicago, Chicago, IL, USA) for her efforts in coordinating this study, the sleep technologists who performed the overnight polysomnograms, the laboratory technicians who drew the labs and performed the analyses, and finally the children and parents for their participation in this study.

\section{References}

1 Ogden CL, Carroll MD, Kit BK, et al. Prevalence of obesity and trends in body mass index among US children and adolescents, 1999-2010. JAMA 2012; 307: 483-490.

2 Fagot-Campagna A, Pettitt DJ, Engelgau MM, et al. Type 2 diabetes among North American children and adolescents: an epidemiologic review and a public health perspective. J Pediatr 2000; 136: 664-672.

3 Weiss R, Dziura J, Burgert TS, et al. Obesity and the metabolic syndrome in children and adolescents. $N$ Engl J Med 2004; 350: 2362-2374.

4 Juonala M, Magnussen CG, Berenson GS, et al. Childhood adiposity, adult adiposity, and cardiovascular risk factors. N Engl J Med 2011; 365: 1876-1885.

Wing RR, Phelan S. Long-term weight loss maintenance. Am J Clin Nutr 2005; 82: Suppl., 222S-225S.

Young T, Skatrud J, Peppard PE. Risk factors for obstructive sleep apnea in adults. JAMA 2004; 291: 2013-2016.

Arens R, Muzumdar H. Childhood obesity and obstructive sleep apnea syndrome. J Appl Physiol 2010; 108: 436-444.

8 Canapari CA, Hoppin AG, Kinane TB, et al. Relationship between sleep apnea, fat distribution, and insulin resistance in obese children. J Clin Sleep Med 2011; 7: 268-273.

9 de la Eva RC, Baur LA, Donaghue KC, et al. Metabolic correlates with obstructive sleep apnea in obese subjects. J Pediatr 2002; 140: 654-659.

10 Tsaoussoglou M, Bixler EO, Calhoun S, et al. Sleep-disordered breathing in obese children is associated with prevalent excessive daytime sleepiness, inflammation, and metabolic abnormalities. J Clin Endocrinol Metab 2010; 95: 143-150.

11 Tauman R, O'Brien LM, Ivanenko A, et al. Obesity rather than severity of sleep-disordered breathing as the major determinant of insulin resistance and altered lipidemia in snoring children. Pediatrics 2005; 116: e66-e73.

12 Ogden CL, Kuczmarski RJ, Flegal KM, et al. Centers for Disease Control and Prevention 2000 growth charts for the United States: improvements to the 1977 National Center for Health Statistics version. Pediatrics 2002; 109: 45-60.

13 Barlow SE, Expert C. Expert committee recommendations regarding the prevention, assessment, and treatment of child and adolescent overweight and obesity: summary report. Pediatrics 2007; 120: Suppl. 4, S164-S192. 
14 American Academy of Sleep Medicine. The AASM Manual for the Scoring of Sleep and Associated Events: Rules, Terminology and Technical Specifications. Westchester, American Academy of Sleep Medicine, 2007.

15 Ridker P, Rifai N, Cook NR, et al. Non-HDL cholesterol, apolipoproteins A-I and B100, standard lipid measures, lipid ratios, and CRP as risk factors for cardiovascular disease in women. JAMA 2005; 294: 326-333.

16 Manninen V, Tenkanen L, Koskinen P, et al. Joint effects of serum triglyceride and LDL cholesterol and HDL cholesterol concentrations on coronary heart disease risk in the Helsinki Heart Study. Implications for treatment. Circulation 1992; 85: 37-45.

17 Di Bonito P, Valerio G, Grugni G, et al. Comparison of non-HDL-cholesterol versus triglycerides-to-HDL-cholesterol ratio in relation to cardiometabolic risk factors and preclinical organ damage in overweight/obese children: the CARITALY study. Nutr Metab Cardiovasc Dis 2015; 25: 489-494.

18 Matthews DR, Hosker JP, Rudenski AS, et al. Homeostasis model assessment: insulin resistance and beta-cell function from fasting plasma glucose and insulin concentrations in man. Diabetologia 1985; 28: 412-419.

19 Yeckel CW, Weiss R, Dziura J, et al. Validation of insulin sensitivity indices from oral glucose tolerance test parameters in obese children and adolescents. J Clin Endocrinol Metab 2004; 89: 1096-1101.

20 Madeira IR, Carvalho CN, Gazolla FM, et al. Ponto de corte do indice Homeostatic Model Assessment for Insulin Resistance (HOMA-IR) avaliado pela curva Receiver Operating Characteristic (ROC) na deteccao de sindrome metabolica em criancas pre-puberes com excesso de peso. [Cut-off point for homeostatic model assessment for insulin resistance (HOMA-IR) index established from receiver operating characteristic (ROC) curve in the detection of metabolic syndrome in overweight pre-pubertal children.] Arq Bras Endocrinol Metab 2008; 52: 1466-1473.

21 Ascaso JF, Pardo S, Real JT, et al. Diagnosing insulin resistance by simple quantitative methods in subjects with normal glucose metabolism. Diabetes Care 2003; 26: 3320-3325.

22 Tauman R, O'Brien LM, Holbrook CR, et al. Sleep pressure score: a new index of sleep disruption in snoring children. Sleep 2004; 27: 274-278.

23 Koren D, Levitt Katz LE, Brar PC, et al. Sleep architecture and glucose and insulin homeostasis in obese adolescents. Diabetes Care 2011; 34: 2442-2447.

24 Grimaldi D, Beccuti G, Touma C, et al. Association of obstructive sleep apnea in rapid eye movement sleep with reduced glycemic control in type 2 diabetes: therapeutic implications. Diabetes Care 2014; 37: 355-363.

25 Ip MS, Lam B, Ng MM, et al. Obstructive sleep apnea is independently associated with insulin resistance. Am J Respir Crit Care Med 2002; 165: 670-676.

26 Tasali E, Mokhlesi B, Van Cauter E. Obstructive sleep apnea and type 2 diabetes: interacting epidemics. Chest 2008; 133: 496-506.

27 Babu AR, Herdegen J, Fogelfeld L, et al. Type 2 diabetes, glycemic control, and continuous positive airway pressure in obstructive sleep apnea. Arch Intern Med 2005; 165: 447-452.

$28 \mathrm{Li}$ AM, Chan MH, Chan DF, et al. Insulin and obstructive sleep apnea in obese Chinese children. Pediatr Pulmonol 2006; 41: 1175-1181.

29 Tauman R, O’Brien LM, Ivanenko A, et al. Obesity rather than severity of sleep-disordered breathing as the major determinant of insulin resistance and altered lipidemia in snoring children. Pediatrics 2005; 116: e66-e73.

30 Gozal D, Capdevila OS, Kheirandish-Gozal L. Metabolic alterations and systemic inflammation in obstructive sleep apnea among nonobese and obese prepubertal children. Am J Respir Crit Care Med 2008; 177: 1142-1149.

31 Kelly A, Dougherty S, Cucchiara A, et al. Catecholamines, adiponectin, and insulin resistance as measured by HOMA in children with obstructive sleep apnea. Sleep 2010; 33: 1185-1191.

32 Hannon TS, Lee S, Chakravorty S, et al. Sleep-disordered breathing in obese adolescents is associated with visceral adiposity and markers of insulin resistance. Int J Pediatr Obes 2011; 6: 157-160.

33 Goran MI, Ball GDC, Cruz ML. Obesity and risk of type 2 diabetes and cardiovascular disease in children and adolescents. J Clin Endocrinol Metab 2003; 88: 1417-1427.

34 Slyper AH. Childhood obesity, adipose tissue distribution, and the pediatric practitioner. Pediatrics 1998; 102: e4.

35 Dayyat E, Kheirandish-Gozal L, Gozal D. Childhood obstructive sleep apnea: one or two distinct disease entities? Sleep Med Clin 2007; 2: 433-444.

36 Matthews KA, Dahl RE, Owens JF, et al. Sleep duration and insulin resistance in healthy black and white adolescents. Sleep 2012; 35: 1353-1358.

37 Stamatakis KA, Punjabi NM. Effects of sleep fragmentation on glucose metabolism in normal subjects. Chest 2010; 137: 95-101.

38 Carreras A, Zhang SX, Peris E, et al. Effect of resveratrol on visceral white adipose tissue inflammation and insulin sensitivity in a mouse model of sleep apnea. Int J Obes 2015; 39: 418-423.

39 Wang Y, Carreras A, Lee S, et al. Chronic sleep fragmentation promotes obesity in young adult mice. Obesity (Silver Spring) 2014; 22: 758-762.

40 Gharib SA, Khalyfa A, Abdelkarim A, et al. Integrative miRNA-mRNA profiling of adipose tissue unravels transcriptional circuits induced by sleep fragmentation. PLoS One 2012; 7: e37669.

41 Pepin JL, Guillot M, Tamisier R, et al. The upper airway resistance syndrome. Respiration 2012; 83: 559-566. 